\title{
2011 for BJID - Some good news and acknowledgements
}

The Brazilian Journal of Infectious Diseases just received its second impact factor by Journal of Citation Report (JCR), which showed an expressive increment (45\%) in comparison with the first one, obtained in 2010. We would like to share this good news with our editorial board, and thank all the referees that contributed to this great achievement. Our goals to the next years are to maintain an increasing level of quality of published papers, and a fast, efficient, and reliable editorial process. A special thank to the referees that have contributed to the revision of manuscripts submitted to BJID in the year of 2011 (see Appendix on page 621).

We are also glad to publish an extremely relevant reflection of Dr. Evaldo Stanislau Affonso de Araújo regarding hepatitis $C$ therapy. Hepatitis $C$ virus infection is already a major public health concern, and contributes to a significant number of deaths worldwide. Although the conventional therapy to treat such infection is only effective for a subset of patients, the development of new drugs increased the chances of cure, even for patients failing previous therapy with pegylated interferon plus ribavirin. An impressive number of new drugs are being tested and promise to revolutionize the treatment of HCV infection in the near future. In this issue, Dr. Evaldo Stanislau Affonso de Araújo reviews the main aspects of the newly released HCV protease inhibitors, boceprevir and telaprevir, and comments the potential impact of these new drugs on HCV treatment, along with some reflections on its role in our future treatment strategies of this important infection. 